\title{
Early Use of Handgrip Exercise Associated with Dobutamine Stress Echocardiography in Women
}

Isabela de Andrade Lindner, ${ }^{\circledR}$ Patricia Sens de Oliveira, ${ }^{\circledR}$ Caroline de Oliveira Fischer Bacca, ${ }^{\circledR}$ Josie Budag Matsuda, ${ }^{\circledR}$ Franciani Rodrigues da Rocha, ${ }^{\circledR}$ Jeancarlo Visentainer, ${ }^{\circledR}$ Luiz Eduardo Bacca ${ }^{\circledR}$

UNIDAVI - Centro Universitário para o Desenvolvimento do Alto Vale do Itajaí, Rio do Sul, SC - Brazil

\section{Abstract}

Background: Coronary artery disease (CAD) is an important cause of morbidity and mortality in women and requires early diagnosis for defining the appropriate treatment.

Objective: To identify the positive predictive value (PPV) and safety of the early use of handgrip exercise in pharmacological stress echocardiography using dobutamine (early-ECHO) in women.

Methods: Positive ischemic early-ECHO records from 111 women were evaluated from January 2012 to March 2018. Subsequently, the hospital medical records were verified to locate patients who underwent conventional coronary angiography (CCA), and we analyzed the medical conduct adopted for these patients. Statistical analyses were performed using SPSS employing one-way analysis of variance (ANOVA), Fisher's exact test, or Pearson's chi-square test. The level of statistical significance was set at $\mathrm{p}<0.05$ for all analyses.

Results: Four patients (4.4\%) presented serious complications during the examination. Out of 90 patients who underwent CCA, 71 (78.9\%) had CAD. Among these 71 patients, 58 (81.7\%) had severe lesions and 13 (18.3\%) presented moderate CAD. Moreover, CCA did not demonstrate relevant coronary lesions in 19 of the 90 patients (21.1\%). Among patients with severe CAD, 16 (27.6\%) underwent myocardial revascularization surgery; 34 (58.6\%) underwent percutaneous coronary angioplasty; and 08 (13.7\%) had their clinical treatments intensified. The PPV for early-ECHO was $78.9 \%$.

Conclusions: Early-ECHO showed a high PPV for diagnosing myocardial ischemia in women. It presented a low complication rate and provided rapid disease identification, allowing the early treatment of injuries and potentially preventing CAD complications.

Keywords: Stress Echocardiography; Coronary Artery Disease; Women; Predictive Value of Tests; Safety.

\section{Introduction}

Ischemic heart disease is an important public health problem in Brazil. Between 2008 and 2018, 488858 deaths of women due to coronary artery disease (CAD) were reported. ${ }^{1}$ According to the American Heart Association (AHA), approximately 1 in 3 women have some form of cardiovascular disease. The evidence shows that women at risk for CAD are less often referred to appropriate diagnostic tests than men. ${ }^{2}$
Clinical presentations of CAD result from atherosclerosis of the coronary arteries and overt angina syndromes, acute myocardial infarction, ischemic cardiomyopathy, and sudden cardiac death. A major aggravating factor in the

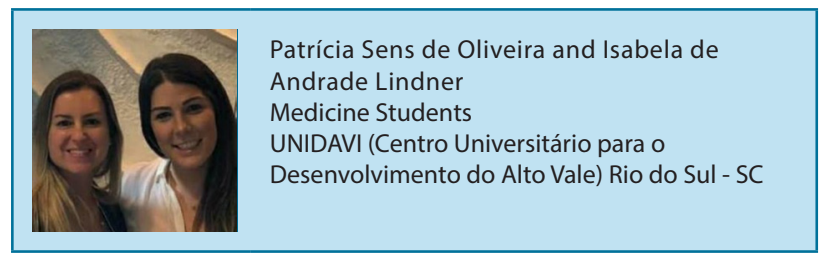


occurrence of CAD is its silent characteristic, which can be perceived at certain effort levels when there is an increase in myocardial oxygen consumption (MVO2). 3,4

In this context, diagnostic tests increase the scope for investigating ischemia. Dobutamine stress echocardiography is an affordable, non-invasive, radiation-free test that allows the assessment of several segments of the left ventricle, making it possible to assess a patient's risk of developing severe CAD. ${ }^{4}$

Currently, the use of the 3-minute dobutamine protocol has become a popular noninvasive technology (NIT), with the addition of atropine at the final stage. ${ }^{5}$ However, atropine may prolong exam time and cause serious side effects. ${ }^{6,7}$

The use of isometric handgrip exercise with dobutamineatropine stress echocardiography (early-ECHO) decreases the time to target heart rate, recovery time, and the overall examination time. ${ }^{8}$ Another advantage of early-ECHO is the reduction of the total dose infusion of dobutamine and its consequent side effects. ${ }^{9}$

Adjunctive isometric exercise in the form of sustained submaximal handgrip dobutamine stress echocardiography without atropine results in a modest increase in MVO2, primarily by an increase in end-systolic wall stress; ${ }^{10}$ it can also significantly lower fractional flow reserve (FFR) values and potentially improve the ability of this test to detect physiologically significant stenosis. ${ }^{11}$

The main goal of this study was to highlight the positive predictive value (PPV) of early-ECHO in detecting CAD in women, emphasizing its safety and applicability.

\section{Methods}

\section{Participants}

This study analyzed the medical records of female patients subjected to pharmacological stress echocardiography with dobutamine with an early protocol (early-ECHO) at a diagnostic imaging center from January 2012 to March 2018.

Secondary data collected from medical records of patients at the imaging center were compared with those of the region's referral hospital in the same period. Data from the center's medical records included age, body mass index (BMI), incidence of complications during the examination (complex ventricular arrhythmia, arterial hypertension above $220 / 110 \mathrm{mmHg}$, and/or angina chest pain), and dobutamine dosages used in the procedure. Data obtained from the hospital's medical records, on the other hand, were used to refer to a conventional coronary angiography (CCA) examination and demonstrate the accuracy of positive early-ECHO results, as well as to quantify blood vessel occlusion and the medical conduct adopted for treating patients.

Inclusion criteria were defined by early-ECHO tests in which a positive ischemia response was observed; exclusion criteria considered tests with a negative ischemia response. At one point, patients who did not undergo CCA at the referral hospital were excluded.

To evaluate the safety and PPV of early-ECHO, we selected the results of 111 patients with positive diagnostic tests. This validation was performed through the search for medical records of these patients at the hospital, followed by an analysis of the CCA examination.

\section{The early-ECHO protocol}

Two-dimensional transthoracic echocardiography with Doppler ultrasound was performed by an experienced certified sonographer-physician using a Phillips Affiniti 70 ultrasound. All patients were examined in the left lateral position.

Dobutamine was diluted in 5\% glycated serum and administered through an infusion pump at doses of 5-10-20-40 $\mathrm{mcg} / \mathrm{kg} / \mathrm{min}$, increasing every $3 \mathrm{minutes}$. Blood pressure (BP) and a 12-lead electrocardiogram (ECG) were acquired every 3 minutes, before increasing the medication dose. At the end of the examination, intravenous beta-blockers (5 mg metoprolol) were administered.

Moreover, in our early-ECHO protocol, isometric exercise (with a handgrip strengthener) was used from the third minute of the examination, when dobutamine dose was $10 \mathrm{mcg} / \mathrm{kg} / \mathrm{min}$. Firstly, the isometric strength exercise was used in the non-dominant hand and, after $\mathrm{BP}$ verification, the isometric strength intensity was increased for both hands in order to reach $85 \%$ of the maximum heart rate (MHR) estimated for a patient of that age or the optimization of a positive ischemia result.

Video records were captured at baseline, with a low dobutamine dose (5 to $10 \mathrm{mcg} / \mathrm{kg} / \mathrm{min}$ ), at peak stress ( $85 \%$ of $\mathrm{MRH})$, and in the recovery phase (after using the beta-blocker). Images were selected from 4 different echocardiographic views: parasternal long axis, parasternal short axis, apical 4-chamber, and apical 2-chamber.

The early-ECHO protocol was first suggested by Yao and colleagues in $2003^{8}$ and was adapted to the patients in this study as shown in Figure 1. 


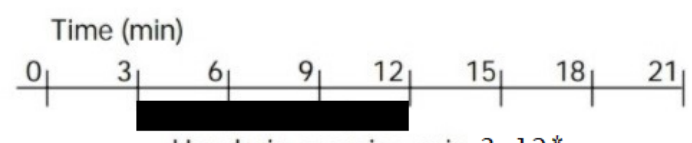

Handgrip exercise, min 3-12*

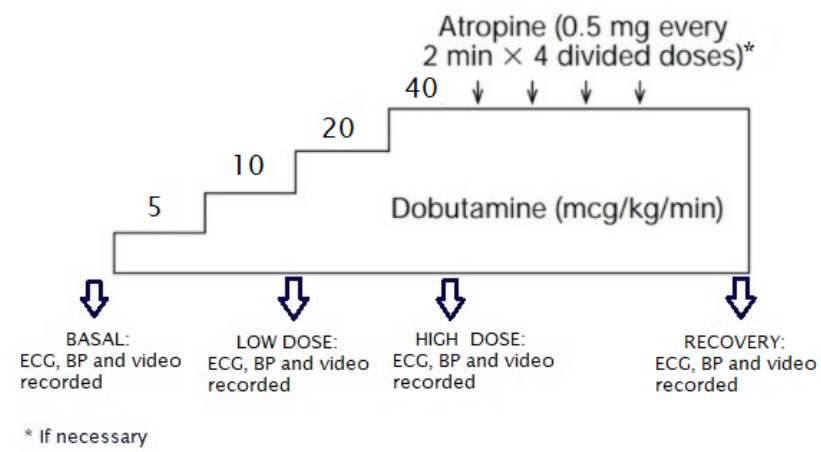

Figure 1 - Early pharmacological stress echocardiogram protocol. Adapted from Yao and colleagues, $2003 .{ }^{8}$ ECG: electrocardiogram; BP: blood pressure.

The presence of ischemia (positive test) was defined as the development of a new wall motion abnormality or worsening of an existing one during stress (hypokinesia, akinesia, or dyskinesia). When the examination demonstrated precocious contractile change, it was interrupted even without reaching the recommended MHR. For interpretive analyses, the left ventricle was divided into 17 segments as recommended by the American Society of Echocardiography. ${ }^{12}$

\section{CCA}

CCA still plays a pivotal role in the invasive imaging of coronary arteries. Despite rapid developments in noninvasive imaging, the temporal and spatial resolution of coronary angiography is unsurpassed and will remain as the road map for cardiology interventionalists and cardiac surgeons for performing revascularization. ${ }^{13}$

The purpose of CCA is to evaluate the coronary anatomy and the degree of luminal obstruction of the coronary arteries. ${ }^{14}$ Angiographically normal or near-normal coronary arteries are more common among women, who are more likely than men to have myocardial ischemia due to microvascular disease. ${ }^{15}$

The degree of coronary stenosis has been determined by the AHA Guideline as: negative (without coronary lesion); discrete (less than $50 \%$ of stenosis); moderate (between $50 \%$ and $70 \%$ of luminal narrowing); and severe (more than $70 \%$ of luminal narrowing). ${ }^{16}$

\section{Statistical analysis}

The extracted data were organized using Microsoft Excel and then transferred to the SPSS software version 22.0, which was used for statistical analyses.

The Komogorov-Smirnov normality test was initially performed for quantitative variables. Given the normality of the findings, a one-way analysis of variance (ANOVA) parametric test was selected for comparing results and verifying statistical inferences. A Tukey's post hoc test was not used because no statistically significant difference was observed.

Quantitative variables are presented as mean values \pm standard deviations. Qualitative variables are presented by n (absolute number) and \% (percentage), and Fisher's exact test (if more than $20 \%$ of the expected value $<5$ ) or Pearson's chi-squared test (if expected frequency $>5$ ) were used as measures of association. In case of a statistically significant difference, the adjusted residuals analysis was used. In this analysis, statistical significance was considered where the frequency was higher (residual equal to or higher than +1.96) and where the frequency was lower (residual equal to or less than -1.96).

In order to determine PPV, the following formula was used: PPV $=$ True Positive / (True Positive + False Positive). A p-value $\alpha=0.05$ ( $\mathrm{p}<0.05)$ was adopted as the level of statistical significance in all analyses. 


\section{Results}

\section{Patient population}

Out of 111 selected patients, 21 medical records were excluded since the CCA examinations had not been performed at the hospital. A total of 90 female patients met all inclusion criteria. The association between CCA outcomes and the variables analyzed in the study is shown in Table 1.
The mean age was $66.59 \pm 9.86$ years $(\mathrm{p}=0.91)$, ranging from 43 to 98 years. No significant difference was observed in the patients' ages and the presence of CAD. Considering the BMI of these women, 37 (41.1\%) were classified as overweight.

Furthermore, out of 90 patients who underwent CCA, $71(78.9 \%)$ presented CAD; among these 71 patients, $13(18.3 \%)$ had moderate CAD and 58 (81.7), severe CAD. Concerning all 90 patients, $19(21.1 \%)$ had negative tests, without significant

Table 1 - Catheterization outcomes (conventional coronary angiography, CCA) compared with the variables analyzed before and during the examination $(n=90)$

\begin{tabular}{|c|c|c|c|c|c|}
\hline Variables & $\begin{array}{c}\text { Negative } \\
\text { n (\%) }\end{array}$ & $\begin{array}{l}\text { Mild stenosis } \\
\text { n (\%) }\end{array}$ & $\begin{array}{c}\text { Severe stenosis } \\
\text { n (\%) }\end{array}$ & $\begin{array}{l}\text { Overall } \\
\text { n (\%) }\end{array}$ & $\mathrm{p}$ \\
\hline CCA & $19(21.1)$ & $13(14.4)$ & $58(64.4)$ & $90(100.0)$ & - \\
\hline PPV & - & - & - & 78.9 & - \\
\hline AGE & $65.95 \pm 12.72$ & $65.30 \pm 11.20$ & $66.55 \pm 8.57$ & $66.59 \pm 9.86$ & $0.91 \mathrm{a}$ \\
\hline \multicolumn{6}{|l|}{ Body mass index } \\
\hline Low weight & $1(14.3)$ & $1(14.3)$ & $5(71.4)$ & $7(100.0)$ & \multirow{5}{*}{$0.89 \mathrm{~b}$} \\
\hline Normal weight & $7(25.0)$ & $5(17.9)$ & $16(57.1)$ & $28(100.0)$ & \\
\hline High weight & $7(18.9)$ & $4(10.8)$ & $26(70.3)$ & $37(100.0)$ & \\
\hline Obesity I & $2(40.0)$ & $2(15.4)$ & $9(69.2)$ & $13(100.0)$ & \\
\hline Obesity II & $2(40.0)$ & $1(10.0)$ & $2(40.0)$ & $5(100.0)$ & \\
\hline \multicolumn{6}{|l|}{ Complications } \\
\hline None & $17(18.9)$ & $13(15.1)$ & $56(65.1)$ & $86(100.0)$ & \multirow{5}{*}{$0.45 b$} \\
\hline Hypertension (220/110 mmHg) & $1(100.0)$ & $0(0.00)$ & $0(0.00)$ & $1(100.0)$ & \\
\hline Complex ventricular arrhythmia & $1(100.0)$ & $0(0.00)$ & $0(0.00)$ & $1(100.0)$ & \\
\hline Typical chest pain & $0(0.00)$ & $0(0.00)$ & $1(100.0)$ & $1(100.0)$ & \\
\hline Arrhythmia and typical chest pain & $0(0.00)$ & $0(0.00)$ & $1(100.0)$ & $1(100.0)$ & \\
\hline \multicolumn{6}{|l|}{ Arrhythmia type } \\
\hline None & $13(21.7)$ & $8(13.3)$ & $39(65.0)$ & $60(100.0)$ & \multirow{4}{*}{$0.65 b$} \\
\hline SVES & $0(0.00)$ & $2(25.0)$ & $6(75.0)$ & $8(100.0)$ & \\
\hline VES & $6(28.6)$ & $3(14.3)$ & $12(57.1)$ & $21(100.0)$ & \\
\hline NSVT & $0(0.00)$ & $0(0.00)$ & $1(100.0)$ & $1(100.0)$ & \\
\hline \multicolumn{6}{|l|}{ Medical procedure after CCA } \\
\hline PTCA & - & - & $34(58.6)$ & - & \\
\hline MRS & - & - & $16(27.6)$ & - & \multirow{2}{*}{ - } \\
\hline Pharmacological treatment & - & - & $8(13.8)$ & - & \\
\hline
\end{tabular}

Statistical methods: a: one-way analysis of variance (ANOVA); b: Fisher's exact test. 
stenosis. The PPV of the early-ECHO examination in women was $78.9 \%$.

Only 4 patients $(4.4 \%)$ had serious complications during the examination, determined as complex ventricular arrhythmias, hypertension (above 220/120 $\mathrm{mmHg}$ ), or typical chest pain. It is also significant that $60(66.7 \%)$ participants did not have arrhythmias during the examination, and from these 60, 39 (65\%) had severe CAD. In the examinations of 58 patients with severe CAD, $06(10.3 \%)$ had supraventricular extrasystole (SVES), $12(20.7 \%)$ had ventricular extrasystole (VES), and 1 (1.7\%) had non-sustained ventricular tachycardia (NSVT).

Regarding the medical conduct for patients with severe CAD, 34 (58.6\%) underwent percutaneous transluminal coronary angioplasty; 16 (27.6\%) underwent myocardial revascularization surgery; and $8(13.8 \%)$ had their clinical treatment intensified.

The association between variables analyzed before and during the examination with a CAD predictor considering patients aged older than 60 years is shown in Table 2 . Additionally, 25 (27.7\%) participants aged $<59$ years and $65(72.2 \%)$ aged $>60$ years were observed. It is noteworthy that women who were younger than 59 years old were more likely to have class 1 obesity (defined as a BMI between 30.0 and $34.9 \mathrm{~kg} / \mathrm{m}^{2}$ ), being represented by 8 patients $(32.0 \%)$ with a statistically significant difference $(\mathrm{p}=0.02)$ and adjusted residues (ra) of 3.1 confirming significance. Furthermore, when grouping overweight patients and those with grade 1 obesity, we observed that $70 \%$ of them presented severe CAD at CCA.

Another characteristic found during the examination was the lower need for high dobutamine doses in women over the age of 60 years. Nearly $81 \%$ of older patients reached the goal of the examination with a lower dose of dobutamine while $44 \%$ of younger patients (< 59 years) required a high dose of this medication, with a statistically significant difference $(p=0.01)$ and $r a=2.5$ confirming the significance.

\section{Discussion}

Stress echocardiography is an established technique for assessing the extent and severity of CAD. The combination of echocardiography with physical, pharmacological, or electrical stress allows the detection of myocardial ischemia with excellent accuracy. This NIT provides diagnostic and prognostic accuracy that is similar to that of radionuclide stress perfusion imaging or magnetic resonance, but at a substantially lower cost and without environmental impact and hazards to the patient and physician. ${ }^{17}$

The present study demonstrated a low rate $(4.4 \%)$ of relevant complications (defined as complex ventricular arrhythmias, arterial hypertension, and/or typical chest pain), which was different from the literature. Abreu et al. indicated an occurrence of typical chest pain in $53.8 \%$ of positive tests in octogenarians and complex arrhythmias, occurring in $6.4 \%$ of the examinations of patients aged 80 years or older. The frequency of extrasystoles varied between $27.6 \%$ in patients aged younger than 60 years and $47.8 \%$ in patients aged 80 years or older. ${ }^{18}$ The finding rate of non-complex arrhythmias in patients with severe CAD was $10.3 \%$ for SVES and $20.7 \%$ for VES. The early use of isometric exercise in our early-ECHO protocol may have been a relevant factor for reducing test duration, as already demonstrated by Yao and colleagues, ${ }^{8}$ thus leading to a lower rate of adverse events was observed.

Regarding the use of medications during the examination, a significant portion of older patients (> 60 years) did not require high dobutamine doses. This was shown by Secnus and Marwick in 1997, when they found that women had a higher heart rate than men both at rest and at the end of ECHO, in addition to the fact that fewer women needed atropine. ${ }^{19}$ Abreu and colleagues also showed that octogenarians (mainly women) needed less medication during the exam, which may be due to less vagal activity and/or greater sensitivity to dobutamine in this population. ${ }^{18}$

Data showed that women aged 59 years and younger were more predisposed to having class 1 obesity. In the obese patient, the blood volume and capillary network are increased. In order to compensate for this, the cardiac output is increased according to the relation between excess weight and ideal weight. There is also an increase in oxygen consumption by the metabolism of adipose tissue, with most of the cardiac output destined to supply these cells. Moreover, the intensity of these changes is proportional to the time since the installation of this hemodynamic situation, and this pattern has been shown to be reversible with weight reduction. ${ }^{20}$

According to Herszkowicz et al., ${ }^{20}$ a population of obese women, without any clinical alterations or cardiovascular complications, showed a trend of higher values of systolic arterial pressure, myocardial mass, and left ventricular wall circumference stress, as well as a trend of early alterations of global diastolic function. ${ }^{20}$ 
Table 2 - Association between the outcome of patients aged 60 years or older and the variables analyzed before and during the examination $(n=90)$

\begin{tabular}{|c|c|c|c|c|}
\hline \multirow{2}{*}{ Variables } & Age $\leq 59$ years old & Age $\geq 60$ years old & \multirow{2}{*}{ n (\%) } & \multirow{2}{*}{$\mathrm{p}$} \\
\hline & $n(\%)(n=25)$ & $n(\%)(n=65)$ & & \\
\hline
\end{tabular}

Body mass index

Low weight

Normal weight

High weight

Obesity I

Obesity II

Basal segment analysis

Normal

Dysfunction

Low dose segment analysis

Normal

Dysfunction

Use of high dose

No

Yes

High dose segment analysis

Normal

Dysfunction

Complications

None

Hypertension (220/110 mmHg)

Complex ventricular arrhythmia

Typical chest pain

Arrhythmia and typical chest pain

Arrhythmia type

None

SVES

VES

NSVT

Medical procedure after CCA

PTCA

MRS

Pharmacological treatment
$9(81.8)$

$24(96.0)$

$1(4.0)$

$$
\begin{gathered}
0(0.0) \\
5(20.0) \\
10(40.0) \\
8(32.0)^{\mathrm{ra}} \\
2(8.0)
\end{gathered}
$$

18 (72.0)

$7(28.0)$

$13(52.0)$

$12(48.0)$

14 (56.0)

$11(44.0)^{\mathrm{ra}}$

$52(80.0)^{\mathrm{ra}}$

13 (20.0)

54 (83.1)

11 (16.9)

37 (56.9)

28 (43.1)

$0(0.0)$

$0(0.0)$

$0(0.0)$

21 (84.0)

$2(8.0)$

2 (8.0)

$0(0.0)$

$$
\begin{aligned}
& 7(28.0) \\
& 3(12.0)
\end{aligned}
$$

2 (8.0)
5 (38.5)

7 (29.2)

17 (70.8)

$0.39^{\mathrm{b}}$

8 (61.5)

62 (95.4)

86 (95.6)

1 (1.1)

1 (1.1)

$0.63^{\mathrm{b}}$

1 (1.1)

1 (1.1)

1 (1.5)

40 (61.5)

$61(67.8)$

$6(9.2)$

18 (27.7)

22 (22.2)

$0.15^{\mathrm{b}}$

1 (1.5)

1 (1.1)

34 (37.8)

16 (17.8)

$0.28^{\mathrm{b}}$<smiles>CCCC</smiles>

$0.81^{\mathrm{a}}$

$0.03^{\mathrm{a}} \mathrm{ra}=2.3$

Statistical methods: $a$ : Pearson's chi-squared test; $b$ : Fisher's exact test.

SVES: supraventricular extrasystole; VES: ventricular extrasystole; NSVT: non-sustained ventricular tachycardia; CCA: conventional coronary angiography; PTCA: percutaneous transluminal coronary angioplasty; MRS: myocardial revascularization surgery. 
Furthermore, the use of preventive measures against obesity in women, as well as the early identification of ventricular remodeling, has become an important factor in the fight against cardiovascular diseases in the female population.

In 2012, a comparative effectiveness review evaluated the diagnostic accuracy and risks of NIT in women with suspected symptoms of CAD. This review showed that ECHO provided a sensitivity of $79 \%$ and a specificity of $83 \%$ when compared with CCA. ${ }^{21}$

Our analyses showed that the PPV of early-ECHO in women was $78.9 \%$. However, this analysis does not include the probability of pre-test CAD among patients, as well as the clinical aspects of thoracic pain presented by them. Pasierski et al., ${ }^{22}$ reported that, in hypertensive patients with angina, the specificity and PPV of exercise echocardiography were $96 \%$ and $97 \%$. They also demonstrated that the advantage of stress echocardiography over the ECG stress test was more obvious in hypertensive women than in hypertensive men. ${ }^{22}$

Tong and Douglas reported the sensitivity and specificity of exercise echocardiography testing in women to be $91 \%$ and $80 \%$, respectively. These data suggest that exercise echocardiography may be the diagnostic test of choice in women. From a cost-effectiveness point of view, these authors believe that most women should undergo exercise echocardiography as the initial diagnostic test, since its diagnostic accuracy is much higher than that of ECG stress testing. ${ }^{23}$

The prevalence of CAD in the studied population was $78.9 \%$, and in cases of severe CAD (>70\% of stenosis), $27.6 \%$ of the patients underwent myocardial revascularization surgery; $58.6 \%$ underwent coronary angioplasty; and $13.8 \%$ received optimized clinical treatment. Heupler et al., ${ }^{24}$ demonstrated that exercise echocardiography provided incremental prognostic information over exercise ECG in populations with mixed genders or mixed pre-test CAD probability. ${ }^{24}$ The incremental value of stress echocardiography over stress electrocardiography in a low-risk but mixed-gender population was demonstrated over 2 decades ago. ${ }^{25}$

Cortigiani et al. ${ }^{26}$ found that echocardiographic evidence of ischemia on dobutamine or dipyridamole stress echocardiography was the only independent predictor of hard events in a group of 456 women who were not known to have CAD. ${ }^{26}$ Supporting this analysis, Davar et al. ${ }^{27}$ showed that positive stress echocardiography was the only independent predictor of future cardiac events, with a relative risk of 8.9 (95\% confidence interval 1.0 to $76.5, p=0.04$ ). The cumulative event-free survival rate 38 months after stress echocardiography was $98.8 \%$ for patients with negative stress echocardiography results and $50.7 \%$ for patients with positive results. ${ }^{27}$

Moreover, in an earlier study, exerciseechocardiography was found to be the optimal method of diagnosing CAD in women. ${ }^{28}$ Recommendations from the European Society of Cardiology suggest the preferential use of non-ionizing imaging techniques in highly vulnerable patients such as younger women. ${ }^{29}$

\section{Conclusions}

Early-ECHO is a test with a low complication rate that shows a high PPV for the diagnosis of myocardial ischemia in women, providing fast identification of this disease with early treatment of injuries and preventing potential major complications and/or death due to CAD.

Finally, more studies should be conducted in order to recommend the early-ECHO protocol as a NIT in the assessment of CAD in women.

\section{Author contributions}

Acquisition of data: Fischer Bacca CO, Lindner IA, Oliveira, PS. Analysis and interpretation of the data: Lindner IA, Rocha, FR, Matsuda JB. Statistical analysis: Lindner, IA, Rocha, FR. Writing of the manuscript: Fischer Bacca CO, Lindner IA, Oliveira PS, Rocha, FR, Bacca LE. Critical revision of the manuscript for intellectual content: Visentainer, J.

\section{Potential Conflict of Interest}

No potential conflict of interest relevant to this article was reported.

\section{Sources of Funding}

There were no external funding sources for this study.

\section{Study Association}

This study is not associated with any thesis or dissertation work. 


\section{Ethics approval and consent to participate}

This study was approved by the Ethics Committee of the UNIDAVI (Centro Universitário para o Desenvolvimento do Alto Vale do Itajaí) under the protocol number CAEE:
9103718800005676 . All the procedures in this study were in accordance with the 1975 Helsinki Declaration, updated in 2013. Informed consent was obtained from all participants included in the study.

\section{References}

1. Brasil. Ministério da Saúde. Banco de dados do Sistema Único de Saúde - DATASUS [Internet]. Brasília (DF); 2020 [Cited 2020 Dec 21]. Available from: http://tabnet.datasus.gov.br/cgi/tabcgi.exe?sim/cnv/obt10uf.def..

2. Mieres JH, Shaw LJ, Arai A, Budoff MJ, Flamm SD, Hundley WG, et al. Role of noninvasive testing in the clinical evaluation of women with suspected coronary artery disease: consensus statement from the Cardiac Imaging Committee, Council on Clinical Cardiology, and the Cardiovascular Imaging and Intervention Committee, Council on Cardiovascular Radiology and Intervention, American Heart Association. Circulation. 2005;111(5):682-96. doi: 10.1161/01.CIR.0000155233.67287.60.

3. Moreira MCV, Montenegro ST, Paola AAV, editors. Livro-texto da Sociedade Brasileira de Cardiologia. 2nd ed. Barueri: Manole; 2015

4. Camarozano AC, Weitzel LH. Ecocardiografia de estresse e contraste. 2nd ed. Rio de Janeiro: Revinter; 2013.

5. Camarozano AC, Siqueira-Filho AG, Weitzel LH, Resende P, Noé RA The effects of early administration of atropine during dobutamine stress echocardiography: advantages and disadvantages of early dobutamine-atropine protocol. Cardiovasc Ultrasound. 2006;4:17. doi: 10.1186/1476-7120-4-17

6. Lewandowski TJ, Armstrong WF, Bach DS. Reduced test time by early identification of patients requiring atropine during dobutamine stress echocardiography. J Am Soc Echocardiogr. 1998;11(3):236-42. doi: 10.1016/s0894-7317(98)70085-9.

7. Tsutsui JM, Osório AF, Lario FA, Fernandes DR, Sodre G, Andrade JL, et al. Comparison of safety and efficacy of the early injection of atropine during dobutamine stress echocardiography with the conventional protocol. Am J Cardiol. 2004;94(11):1367-72. doi: 10.1016/j. amjcard.2004.07.141.

8. Yao SS, Moldenhauer S, Sherrid MV. Isometric handgrip exercise during dobutamine-atropine stress echocardiography increases heart rate acceleration and decreases study duration and dobutamine and atropine dosage. Clin Cardiol. 2003;26(5):238-42. doi: 10.1002/clc.4960260509.

9. Dattilo G, Patanè S, Zito C, Lamari A, Tulino D, Marte F, et al. Handgrip exercise associated with dobutamine stress echocardiography. Int J Cardiol. 2010;143(3):298-301. doi: 10.1016/j.ijcard.2009.03.016.

10. Khan IA, Otero FJ, Font-Cordoba J, McCulloch M, Sheahan RG, Parmar R, et al. Adjunctive handgrip during dobutamine stress echocardiography: invasive assessment of myocardial oxygen consumption in humans. Clin Cardiol. 2005;28(7):349-52. doi: 10.1002/clc.4960280709.

11. Katritsis DG, Korovesis S, Karvouni E, Giazitzoglou E, Karabinos I, Tzanalaridou E, et al. Handgrip-enhanced myocardial fractional flow reserve for assessment of coronary artery stenoses. Am Heart J. 2006;151(5):1107.e1-7. doi: 10.1016/j.ahj.2005.09.023.

12. Pellikka PA, Arruda-Olson A, Chaudhry FA, Chen MH, Marshall JE, Porter TR, et al. Guidelines for performance, interpretation, and application of stress echocardiography in ischemic heart disease: from the American Society of Echocardiography. J Am Soc Echocardiogr. 2020;33(1):1-41.e8. doi: 10.1016/j.echo.2019.07.001

13. Pijls NH, Sels JW. Functional measurement of coronary stenosis. J Am Coll Cardiol. 2012;59(12):1045-57. doi: 10.1016/j.jacc.2011.09.077.

14. Scanlon PJ, Faxon DP, Audet AM, Carabello B, Dehmer GJ, Eagle KA, et al. ACC/AHA Guidelines for Coronary Angiography: executive summary and recommendations. A report of the American College of Cardiology/ American Heart Association Task Force on Practice Guidelines

(Committee on Coronary Angiography) developed in collaboration with the Society for Cardiac Angiography and Interventions. Circulation. 1999;99(17):2345-57. doi: 10.1161/01.cir.99.17.2345.

15. Dehmer GJ, Weaver D, Roe MT, Milford-Beland S, Fitzgerald S, Hermann A, et al. A contemporary view of diagnostic cardiac catheterization and percutaneous coronary intervention in the United States: a report from the CathPCI Registry of the National Cardiovascular Data Registry, 2010 through June 2011. J Am Coll Cardiol. 2012;60(20):2017-31. doi: 10.1016/j. jacc.2012.08.966.

16. Fihn SD, Blankenship JC, Alexander KP, Bittl JA, Byrne JG, Fletcher BJ, et al. 2014 ACC/AHA/AATS/PCNA/SCAI/STS focused update of the guideline for the diagnosis and management of patients with stable ischemic heart disease: a report of the American College of Cardiology/ American Heart Association Task Force on Practice Guidelines, and the American Association for Thoracic Surgery, Preventive Cardiovascular Nurses Association, Society for Cardiovascular Angiography and Interventions, and Society of Thoracic Surgeons. J Thorac Cardiovasc Surg. 2015;149(3):e5-23. doi: 10.1016/j.jtcvs.2014.11.002.

17. Sicari R, Cortigiani L. The clinical use of stress echocardiography in ischemic heart disease. Cardiovasc Ultrasound. 2017;15(1):7. doi: 10.1186/ s12947-017-0099-2.

18. Abreu JS, Diógenes TC, Farias AG, Morais JM, Paes JN Jr. Safety and feasibility of dobutamine-atropine stress echocardiography in octogenarian patients. Arq Bras Cardiol. 2005;85(3):198-204. doi: 10.1590/ s0066-782x2005001600009.

19. Secknus MA, Marwick TH. Influence of gender on physiologic response and accuracy of dobutamine echocardiography. Am J Cardiol. 1997;80(6):721-4. doi: 10.1016/s0002-9149(97)00502-x.

20. Herszkowicz N, Barbato A, Salvi W, Pinheiro D, Pantaleão D, Halpern A, et al. Contribution of Doppler echocardiography to the evaluation of systolic and diastolic function of obese women versus a control group. Arq Bras Cardiol. 2001;76(3):189-96. doi: 10.1590/s0066$782 \times 2001000300002$

21. Dolor RJ, Patel MR, Melloni C, Chatterjee R, McBroom AJ, Musty MD, et al. Noninvasive technologies for the diagnosis of coronary artery disease in women. Rockville (MD): Agency for Healthcare Research and Quality (US); 2012.

22. Pasierski T, Szwed H, Malczewska B, Firek B, Kośmicki M, Rewicki $\mathrm{M}$, et al. Advantages of exercise echocardiography in comparison to dobutamine echocardiography in the diagnosis of coronary artery disease in hypertensive subjects. J Hum Hypertens. 2001;15(11):805-9. doi: 10.1038/sj.jhh.1001265.

23. Tong AT, Douglas PS. Stress echocardiography in women. Cardiol Clin. 1999;17(3):573-82. doi: 10.1016/s0733-8651(05)70097-7.

24. Heupler S, Mehta R, Lobo A, Leung D, Marwick TH. Prognostic implications of exercise echocardiography in women with known or suspected coronary artery disease. J Am Coll Cardiol. 1997;30(2):414-20. doi: 10.1016/s0735-1097(97)00167-8.

25. Colon PJ 3rd, Mobarek SK, Milani RV, Lavie CJ, Cassidy MM, Murgo JP, et al. Prognostic value of stress echocardiography in the evaluation of atypical chest pain patients without known coronary artery disease. Am J Cardiol. 1998;81(5):545-51. doi: 10.1016/s0002-9149(97)00987-9.

26. Cortigiani L, Dodi C, Paolini EA, Bernardi D, Bruno G, Nannini E. Prognostic value of pharmacological stress echocardiography in women 
with chest pain and unknown coronary artery disease. J Am Coll Cardiol. 1998;32(7):1975-81. doi: 10.1016/s0735-1097(98)00477-x.

27. Davar JI, Roberts EB, Coghlan JG, Evans TR, Lipkin DP. Prognostic value of stress echocardiography in women with high ( $>$ or $=80 \%$ ) probability of coronary artery disease. Postgrad Med J. 2001;77(911):573-7. doi: 10.1136/ pmj.77.911.573

28. Marwick TH, Anderson T, Williams MJ, Haluska B, Melin JA, Pashkow F, et al. Exercise echocardiography is an accurate and cost-efficient technique for detection of coronary artery disease in women. J Am Coll Cardiol. 1995;26(2):335-41. doi: 10.1016/07351097(95)80004-z.

29. Kristensen SD, Knuuti J, Saraste A, Anker S, Bøtker HE, Hert SD, et al. 2014 ESC/ESA Guidelines on non-cardiac surgery: cardiovascular assessment and management: The Joint Task Force on non-cardiac surgery: cardiovascular assessment and management of the European Society of Cardiology (ESC) and the European Society of Anaesthesiology (ESA). Eur Heart J. 2014;35(35):2383-431. doi: 10.1093/eurheartj/ehu282. 